\title{
Using Nile Red-Adsorbed Gold Nanoparticles To Locate Glutathione within Erythrocytes
}

\author{
Wei-Lung Tseng, Kun-Hong Lee, and Huan-Tsung Chang* \\ Department of Chemistry, National Taiwan University, Taipei, Taiwan
}

Received April 25, 2005. In Final Form: August 22, 2005

\begin{abstract}
An aqueous solution of Nile Red (NR)-absorbed 32-nm gold nanoparticles (AuNPs) have been used to sense glutathione (GSH). When the NR product is displaced by GSH on the AuNP surface, the fluorescence of the solution increases and the AuNPs aggregate. To determine the concentration and distribution of GSH within erythrocyte cells, a homemade fluorescence and scattering microscope was constructed. This system allows monitoring, within individual cells, of the uptake and transportation of the NRAuNPs and the displacement of the NR product from the NRAuNP surface by GSH. The fluorescence and scattering images clearly indicate the location of GSH inside the cells; these findings are supported by images recorded using 2,3-naphthalenedicarboxaldehyde, which is a highly selective fluorogenic reagent for GSH. Microscopic fluorescence measurements of the NRAuNPs revealed that the GSH concentration inside erythrocyte cells is $1.30 \pm 0.31 \mathrm{mM}$. To confirm this result, lysed erythrocyte cells were analyzed by applying capillary electrophoresis in conjunction with laser-induced fluorescence using NRAuNPs; accordingly, the average GSH concentration in a single erythrocyte cell was determined to be $1.32 \pm 0.06 \mathrm{mM}$.
\end{abstract}

\section{Introduction}

Thiol-containing compounds continue to be of interest because of their important roles in many biological processes, for example, as antioxidants. ${ }^{1}$ Glutathione (GSH) is the major cellular antioxidant that plays a crucial role in maintaining the balance between oxidation and antioxidation. This tripeptide is produced naturally in the body and is the most abundant $(0.5-10 \mathrm{mM})$ intracellular nonprotein thiol. ${ }^{2}$ Oxidation of the reduced GSH, to form oxidized GSH (GSSG), is performed by direct interaction either with free radicals or, more often, with enzymes, such as GSH peroxidase and glutathione transhydrogenase. GSH also plays an important part in cellular detoxification and is required for many aspects of immune response. ${ }^{3}$ Recent evidence suggests that GSH may also be an important factor in brain damage, ${ }^{4}$ glucose homeostasis, ${ }^{5} \mathrm{HIV}$ expression, ${ }^{6}$ and cancer therapy. ${ }^{7}$

Because of the biological and clinical significance of GSH, a variety of approaches have been developed to determine the concentration and/or distribution of GSH in biological substrates such as body fluids, cells, and tissues. ${ }^{8-27}$ High-performance liquid chromatography and

* Address correspondence to this author at the Department of Chemistry, National Taiwan University, 1, Sec. 4, Roosevelt Rd., Taipei, Taiwan 106 (e-mail changht@ntu.edu.tw; fax 011-886-23366-1171).

(1) Wu, G.; Fang, Y. Z.; Yang, S.; Lupton, J. R.; Turner, N. D. J. Nutr. 2004, 134, 489-492. 160.

(2) Kosower, N. S.; Kosower, E. M. Int. Rev. Cytol. 1978, 54, 109-

(3) Santangelo, F. Curr. Med. Chem. 2003, 10, 2599-2610.

(4) Tsakiris, S.; Schulpis, K. H.; Marinou, K.; Behrakis, P.Pharmacol Res. 2004, 49, 475-479.

(5) Barbagallo, M.; Dominguez, L. J.; Tagliamonte, M. R.; Resnick, L. M.; Paolisso, G. Hypertension 1999, 34, 1002-1006.

(6) Yamaguchi, T.; Katoh, I.; Kurata, S. Eur. J. Biochem. 2002, 269, 2782-2788.

(7) Balendiran, G. K.; Dabur, R.; Fraser, D. Cell Biochem. Funct. 2004, 22, 343-352.

(8) Remião, F.; Carmo, H.; Carvalho, F.; Bastos, M. L. Biomed. Chromatogr. 2000, 14, 468-473.

(9) Houze, P.; Gamra, S.; Madelaine, I.; Bousquet, B.; Gourmel, B. J. Clin. Lab. Anal. 2001, 15, 144-153.

(10) Giustarini, D.; Dalle-Donne, I.; Colombo, R.; Milzani, A.; Rossi, R. Free Radical Biol. Med. 2003, 35 1365-1372. capillary electrophoresis (CE) are common tools used to determine the GSH content in biological samples. ${ }^{8-14} \mathrm{CE}$ in conjunction with laser-induced fluorescence (LIF), which provides the advantages of sensitivity, efficiency, rapidity, and the need for only minute sample volumes, is a powerful technique for the determination of GSH within single cells. ${ }^{15,16} \mathrm{~A}$ spectrofluorometric assay, which uses 5-maleimidyl-2-( $m$-methylphenyl)benzoxazole and an electrochemical sensor in conjunction with a redox cofactor (pyrroloquinoline quinone in a polypyrrole matrix), has been demonstrated for the determination of GSH. ${ }^{17,18}$ Although these methods are sensitive and simple, little information is provided regarding the localization of GSH within single cells. The cellular distribution of GSH can be imaged by fluorescence microscopy measurements using GSH-selective fluorescent dyes, including mercury orange, ${ }^{19,20} \quad o$-phthalaldehyde, ${ }^{21}$ monobromobimane (MBB), ${ }^{22-24}$ monochlorobimane (MCB),${ }^{25-27}$ and 2,3-naphthalenedicarboxaldehyde (NDA). ${ }^{16}$ The fluorescent products that are formed from the interaction between the

(11) Raggi, M. A.; Mandrioli, R.; Casamenti, G.; Musiani, D.; Marini, M. Biomed. Chromatogr.1998, 12, 262-266.

(12) O’Brien, K. B.; Esguerra, M.; Miller, R. F.; Bowser, M. T. Anal. Chem. 2004, 76, 5069-5074.

(13) Jin, W.; Li, X.; Gao, N. Anal. Chem. 2003, 75, 3859-3864.

(14) Inoue, T.; Kirchhoff, J. R. Anal. Chem. 2002, 74, 1349-1354.

(15) Hogan, B. L.; Yeung, E. S. Anal. Chem. 1992, 64, 2841-2845.

(16) Orwar, O.; Fishman, H. A.; Ziv, N. E.; Scheller, R. H.; Zare, R. N. Anal. Chem. 1995, 67, 4261-4268.

(17) Liang, S.-C.; Wang, H.; Zhang, Z.-M.; Zhang, X.; Zhang, H.-S. Anal. Chim. Acta 2002, 451, 211-219.

(18) Inoue, T.; Kirchhoff, J. R. Anal. Chem. 2000, 72, 5755-5760.

(19) Pearce, R. K.; Owen, A.; Daniel, S.; Jenner, P.; Marsden, C. D. J. Neural Transm. 1997, 104, 661-677.

(20) Söderdahl, T.; Enoksson, M.; Lundberg, M.; Holmgren, A.; Ottersen, O. P.; Orrenius, S.; Bolcsfoldi, G.; Cotgreave, I. A. FASEB J. 2003, 17, 124-126.

(21) Treumer, J.; Valet, G. Exp. Cell Res. 1986, 163, 518-524

(22) Hedley, D. W.; Chow, S. Cytometry 1994, 15, 349-358.

(23) McNamara, K. P.; Yeung, E. S.; Rosenzweig, N.; Rosenzweig, Z. Anal. Chim. Acta 1997, 356, 75-83.

(24) Sebastia, J.; Cristofol, R.; Martin, M.; Rodriguez-Farre, E.; Sanfeliu, C. Cytometry 2003, 51, 16-25.

(25) Bellomo, G.; Vairetti, M.; Stivala, L.; Mirabelli, F.; Richelmi, P.; Orrenius, S. Proc. Natl. Acad. Sci. U.S.A. 1992, 89, 4412-4416.

(26) Keelan, J.; Allen, N. J.; Antcliffe, D.; Pal, S.; Duchen, M. R. J. Neurosci. Res. 2001, 66, 873-884.

(27) Meyer, A. J.; May, M. J.; Fricker, M. Plant J. 2001, 27, 67-78. 
amino group of these dyes and the sulfhydryl group of GSH have very high quantum yields and display different excitation/emission spectral bands relative to those of the free fluorescent dyes. MCB and NDA have been used to observe the intracellular distribution of GSH in cultured hepatocyte ${ }^{25}$ and neurobiological samples, ${ }^{16}$ including NG 108-15 cells (mouse neuroblastoma $\times$ rat glioma) and primary glial and neuronal cell cocultures (rat hippocampus), respectively. The mean concentrations of GSH in erythrocytes and NG 108-15 cell lysates are 1 and $5 \mathrm{mM}$, respectively. ${ }^{28,29}$ Two common disadvantages of these methods are that (1) these dyes are generally prepared in organic solutions that may disturb the biological function of the cells and (2) these dyes also react with intracellular thiols other than GSH and proteins, which results in a high fluorescence background and poor spatial resolution.

Gold nanoparticles (AuNPs) have emerged as important sensing materials because they display size-dependent optical properties and have an extremely high affinity toward thiols and thiol-modified molecules. ${ }^{30-33}$ Because of their very high extinction coefficient, AuNPs-acting as a quencher-have been applied to determine DNA labeled with fluorophores (donors) on the basis of fluorescence resonance energy transfer (FRET) ${ }^{34,35}$ Recently, we developed an aqueous solution of 32-nm AuNPs-in which Nile Red (NR) was adsorbed to the AuNPs through non-covalent interactions - to sense thiols. ${ }^{33}$ From measurements of optical properties, mass spectral data, and migration times, we found that a new product formed from the reaction of NR on the AuNP surface. By monitoring increases in fluorescence that occurred when this product was displaced by thiols (using NRAuNPs at $\mathrm{pH} 4.0$ and irradiating the solution at $543 \mathrm{~nm}$ ), we calculated the limits of detection (LODs) for homocysteine, cysteine, and GSH to be $15.4,10.9$, and $191.2 \mathrm{nM}$, respectively. In that study, we also observed aggregation of AuNPs at $\mathrm{pH} 4.0$ in the presence of neutral thiols, such as GSH.

Those results suggested to us the possibility of performing fluorescence and scattering measurements by using the NRAuNPs to monitor GSH within single erythrocytes. We constructed a fluorescence and scattering microscopy system and employed this laboratory-made apparatus, in conjunction with NRAuNPs, to provide images of the distribution and concentrations of GSH within erythrocytes. To confirm the results obtained by fluorescence microscopy, we also used CE-LIF to determine the concentrations of GSH in erythrocytes incubated with NRAuNPs or NDA.

\section{Experimental Section}

Chemicals. Sodium tetrachloroaurate(III) dihydrate, bovine serum albumin (BSA), carbonic anhydrase, asparagine, hemoglobulin, cysteine, sodium tetraborate, and glycine were obtained from Sigma (St. Louis, MO). GSH, dimethyl sulfoxide, and trisodium citrate were purchased from Aldrich (Milwaukee, WI). NR was obtained from Acros (Geel, Belgium). NDA was purchased from Tokyo Chemical Industry (Tokyo, Japan) and was prepared in acetonitrile (ACN) prior to use for derivatization of GSH.

(28) Rossi, R.; Milzani, A.; Dalle-Donne, I.; Giustarini, D.; Lusini, L.; Colombo, R.; Simplicio, P. D. Clin. Chem. 2002, 48, 742-753.

(29) Patel, N.; Kumagai, Y.; Unger, S. E.; Fukuto, J. M.; Cho, A. K Chem. Res. Toxicol. 1991, 4, 421-426.

(30) Sandstrom, P.; Boncheva, M.; Akerman, B. Langmuir 2003, 19 7537-7543.

(31) Zhang, F. X.; Han, L.; Israel, L. B.; Daras, J. G.; Maye, M. M.; Ly, N. K.; Zhong, C. J. Analyst 2002, 127, 462-465.

(32) Daniel, M.-C.; Astruc, D. Chem. Rev. 2004, 104, 293-346.

(33) Chen, S.-J.; Chang, H.-T. Anal. Chem. 2004, 76, 3727-3734.

(34) Dubertret, B.; Calame, M.; Libchaber, A. J. Nat. Biotechnol. 2001, 19, 365-370.

(35) Thomas, K. G.; Kamat, P. V.Acc. Chem. Res. 2003, 36, 888-898.

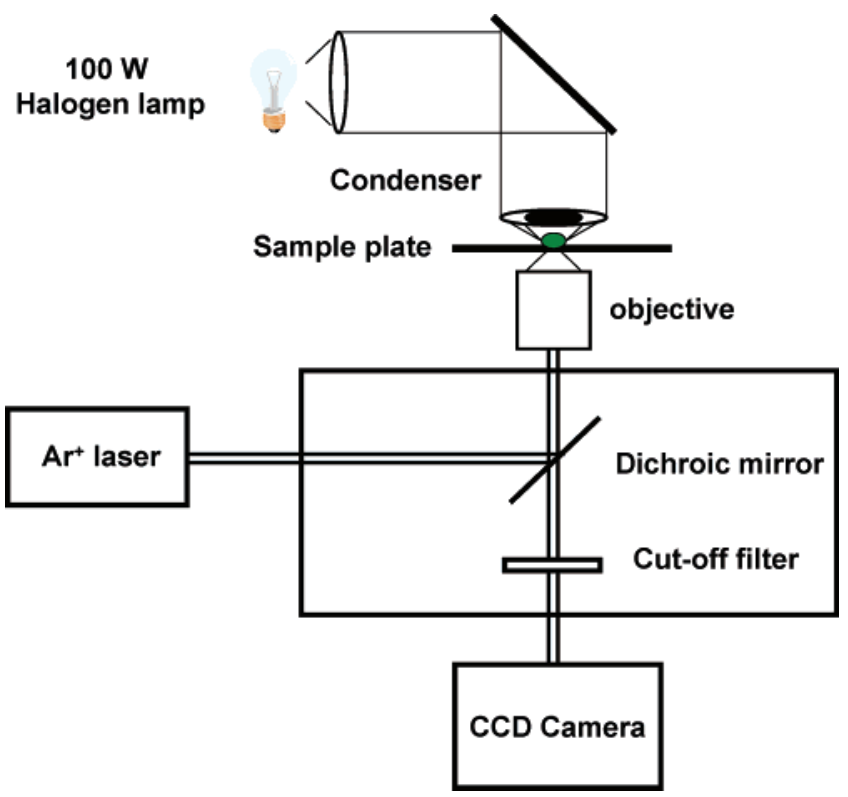

Figure 1. Schematic representation of the scattering and fluorescence microscopy system used for detecting single cells.

Isotonic phosphate-buffered saline (PBS; $\mathrm{pH} 7.4$ ) was prepared by dissolving $\mathrm{Na}_{2} \mathrm{HPO}_{4} \cdot 2 \mathrm{H}_{2} \mathrm{O}(22.05 \mathrm{~g}), \mathrm{NaH}_{2} \mathrm{PO}_{4} \cdot \mathrm{H}_{2} \mathrm{O}(2.07 \mathrm{~g})$, and $\mathrm{NaCl}(4.5 \mathrm{~g})$ in $\mathrm{H}_{2} \mathrm{O}(1.0 \mathrm{~L})$.

Synthesis of 32-nm AuNPs. Trisodium citrate (1\%, $0.5 \mathrm{~mL})$ was added rapidly to an aliquot of $0.01 \% \mathrm{HAuCl}_{4}(50 \mathrm{~mL})$ that was heated under reflux. ${ }^{36,37}$ Heating under reflux was continued for an additional $8 \mathrm{~min}$, during which time the color changed to deep red. The solution was set aside to cool to room temperature; its UV-vis absorption spectrum displayed a surface plasmon resonance (SPR) band at $528 \mathrm{~nm}$. TEM images (not shown) confirmed that the sizes of the AuNPs were $32( \pm 9 \%) \mathrm{nm}$. We note that the as-prepared AuNPs were stable for at least 6 months. The concentration of the original 32-nm AuNP suspension was $\sim 0.27 \mathrm{nM}\left(1.6 \times 10^{11}\right.$ particles $\left./ \mathrm{mL}\right)$, which we denote in this study as $1 \times$.

Preparation of NRAuNPs. A stock solution of NR (1 mM) was prepared in dimethyl sulfoxide. An aliquot of NR solution $(1 \mathrm{mM}, 50 \mu \mathrm{L})$ was added to the $1 \times$ solution of AuNPs $(50 \mathrm{~mL})$, and then the mixture was equilibrated overnight at ambient temperature and pressure. After being subjected to two repeated cycles of centrifugation [at $12000 \mathrm{rpm}$ (relative centrifugal force $=16000 \mathrm{~g}$ ) for $10 \mathrm{~min}$ and washing with citrate buffer $(5 \mathrm{~mL})]$, finally, the precipitates were resuspended in citrate buffer. We denote this as-prepared mixture as an "NRAuNP" solution and, for simplicity, its concentration is presented as $1 \times$. To prepare an NRAuNP solution in PBS buffer, $1 \times$ NRAuNP $(40 \mathrm{~mL})$ was mixed with $100 \mathrm{mM}$ glycine (pH 9.0; $10 \mathrm{~mL})$ before BSA (1 mM, $500 \mu \mathrm{L}$ ) was added. This solution was equilibrated for $1 \mathrm{~h}$ before it was subjected to two centrifugation/washing cycles to remove any excess NR. Centrifugation was conducted at $12000 \mathrm{rpm}$ for $10 \mathrm{~min}$, and PBS $(40 \mathrm{~mL})$ was used to redisperse the sediment. Finally, the sediment was resuspended in PBS buffer. An AmincoBowman fluorometer (ThermoSpectronic, Pittsford, NY) was used to collect the fluorescence spectra of NR and the NRAuNPs in the presence and absence of proteins and amino acids. The emission spectra were recorded while the solution was irradiated at $488 \mathrm{~nm}$.

Fluorescence and Scattering Imaging. An Olympus IX70 inverted microscope (Tokyo, Japan) was used to construct the scattering and fluorescence microscopy system depicted in Figure 1. A 100-W halogen lamp in conjunction with a high-numericalaperture dark-field condenser (NA $=1.2-1.4$; U-DCW, Olympus) was used to illuminate the nanoparticles. An air-cooled $\mathrm{Ar}^{+}$ion laser (model PS9600, Laser Graphics, Dieburg, Germany) having a 1-mW output at $488 \mathrm{~nm}$ was employed to excite the NR product

(36) Frens, G. Nature 1973, 241, 20-22.

(37) Grabar, K. C.; Freeman, R. G.; Hommer, M. B.; Natan, M. J. Anal. Chem. 1995, 67, 735-743. 
and the NDA-GSH derivatives. The fluorescence and scattering signals were collected using a $100 \times(\mathrm{NA}=1.25$; oil immersion $)$ or $40 \times(\mathrm{NA}=0.55)$ objective. To reject scattered light, the fluorescence emissions were passed through RG 590 (in the case of NRAuNP) and RG 570 (in the case of NDA) cutoff filters (Edmund Industrial Optics, Barrington, NJ). The fluorescence images and wide-field images were collected into a digital color camera (Coolpix 5400, Nikon, Tokyo, Japan) attached to the side port of the microscope. The low-cost camera provides images at a resolution of up to $2592 \times 1944$ pixels, which represents a 200 $\times 150 \mu \mathrm{m}$ detection area when using a $40 \times$ objective. This system also allows video images to be recorded at a resolution of $640 \times$ 480 pixels at a rate of $15 \mathrm{frames} / \mathrm{s}$ (with a $33-\mathrm{ms}$ exposure time) for up to $70 \mathrm{~s}$. The motion pictures were recorded as Quick Time files and imported into Macromedia flash (Macromedia, Inc., San Francisco, CA) to obtain a sequence of images. The ImageJ program (http://rsb.info.nih.gov/ij/) was used to analyze the images and calculate the fluorescence intensity.

Calibration Curve for GSH in NRAuNPs. To prepare standard samples of GSH having concentrations in the range from $10 \mu \mathrm{M}$ to $300 \mathrm{mM}, 1 \times \mathrm{NRAuNP}$ solutions $(990 \mu \mathrm{L})$ were spiked with samples of the working solution $(10 \mu \mathrm{L})$. The calibration curve was constructed by a linear regression of the fluorescence intensity of the NR product against the corresponding GSH concentrations over the range of $0.1-10 \mu \mathrm{M}$. The fluorescence of the NR product is quenched, however, by the AuNP aggregates induced by GSH at concentrations $>30 \mu \mathrm{M}$. The relationship between the fluorescence intensity and the concentration of GSH was obtained from the following equation:

$$
F_{0} / F=1+K[\mathrm{GSH}]
$$

where $F_{0}$ is the fluorescence intensity of the NR product in the presence of $30 \mu \mathrm{M} \mathrm{GSH}, F$ is the florescence intensity at different GSH concentrations, and $K$ is the quenching constant. A plot of $F_{0} / F$ versus $[\mathrm{GSH}]$ yields a straight line, the slope of which gives $K$.

Cell Isolation and Loading. Erythrocytes were collected from a healthy male adult donor. A sample of blood ( $20 \mu \mathrm{L})$ was dispensed in PBS (1.0 mL), mixed gently, and then centrifuged at $126 \mathrm{~g}$ for $10 \mathrm{~min}$. The supernatant was discarded into a waste bottle containing $10 \%$ bleach. PBS $(1.0 \mathrm{~mL})$ was then added, and the sample was mixed gently with a pellet. This washing procedure was repeated four times to remove any serum proteins. Finally, the erythrocytes were mixed separately in a solution $(1.0 \mathrm{~mL}$ ) of $1 \times$ NRAuNPs and $10.0 \mu \mathrm{M}$ NDA (both prepared in PBS). The solutions were either used immediately without any other treatment or subjected to three centrifugation/washing cycles to remove any excess NRAuNPs and NDA after equilibrium at ambient temperature and pressure for a certain time (up to $12 \mathrm{~h}$ and $10 \mathrm{~min}$, respectively). After incubation, the samples and cell solutions $(20 \mu \mathrm{L})$ were sandwiched between two cover slips (100- $\mu \mathrm{m}$ thickness) prior to microscopic fluorescence and scattering measurements.

Capillary Electrophoresis. A 5-mW Ar ${ }^{+}$ion laser $(488 \mathrm{~nm})$ was used in the CE-LIF system to excite the analytes. The fluorescence light was collected with a $20 \times$ objective and focused onto a photomultiplier tube (R928, Hamamatsu Photonics K.K. Shizuoka-Ken, Japan). An RG 590 (in the case of NRAuNP) or RG 570 (in the case of NDA) cutoff filter was used to block any scattered light. The fluorescence signal was transferred directly through a $10-\mathrm{k} \Omega$ resistor to a 24 -bit $\mathrm{A} / \mathrm{D}$ interface at $10 \mathrm{~Hz}$ (Borwin, JMBS Developments, Le Fontanil, France) and stored in a PC. The fused-silica capillaries (Polymicro Technologies, Phoenix, AZ) were $40 \mathrm{~cm}$ long and had an i.d. of $75 \mu \mathrm{m}$ (effective length $=30 \mathrm{~cm}$ ). When using NRAuNPs, a sample of erythrocytes $(10 \mu \mathrm{L})$ was mixed with the $1 \times$ NRAuNPs $(990 \mu \mathrm{L} ; \mathrm{pH} 4.0)$. The mixture was subjected to centrifugation at $12000 \mathrm{rpm}$ for $10 \mathrm{~min}$ to remove the pellets and AuNPs. This process also prevents further displacement of the NR product from the NRAuNPs by the action of ACN during the CE separation. When NDA was used, a sample of the erythrocytes $(50 \mu \mathrm{L})$ was mixed with a solution $(450 \mu \mathrm{L})$ of $10 \mathrm{mM}$ sodium tetraborate $\left(\mathrm{pH}^{*} 9.0\right)$ containing $80 \% \mathrm{ACN}$ and $50 \mu \mathrm{M}$ NDA. Before sample injection, the lysed samples that were treated with the NRAuNPs and NDA were diluted by factors of 20 and 2 , respectively, with 10
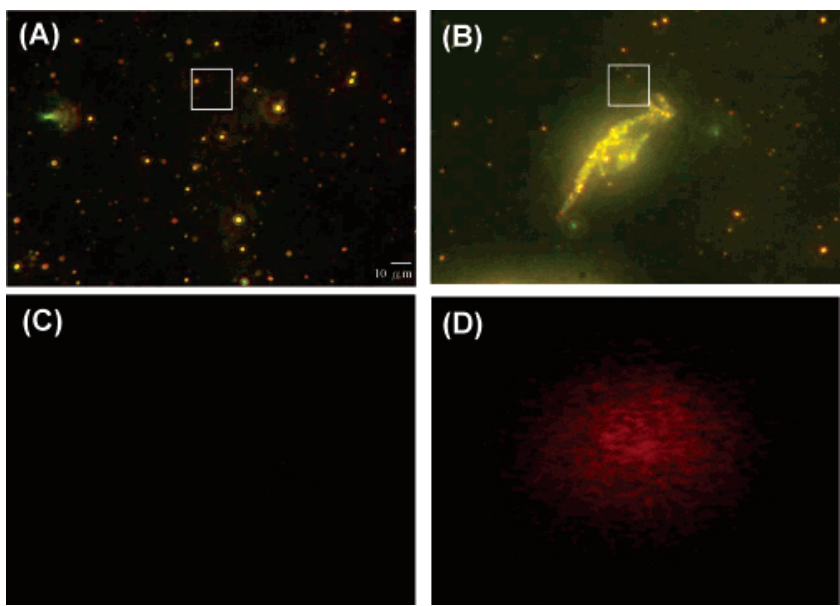

Figure 2. (A, B) Scattering and (C, D) fluorescence images of NRAuNPs in $(A, C)$ the absence and $(B, D)$ the presence of 1.0 mM GSH: exposure times, 0.5 and $4 \mathrm{~s}$ for the scattering and fluorescence measurements, respectively; equilibrium time, 10 min; scattering detection area with a $40 \times$ objective, $200 \mu \mathrm{m} \times$ $150 \mu \mathrm{m}$, which corresponds to 2592 (horizontal) $\times 1944$ (vertical) pixels; fluorescence detection area with a $40 \times$ objective, $10 \mu \mathrm{m}$ $\times 10 \mu \mathrm{m}$. The fluorescence images $(\mathrm{C}, \mathrm{D})$ were recorded in the square areas marked in (A) and (B), respectively.

$\mathrm{mM}$ sodium tetraborate $\left(\mathrm{pH}^{*} 9.0\right)$ containing $25 \%$ ACN. Sample injection was conducted by hydrodynamic means (15-cm height for $15 \mathrm{~s}$ ), and the separation was performed under an applied voltage of $10 \mathrm{kV}$. The capillary was filled with $10 \mathrm{mM}$ sodium tetraborate $\left(\mathrm{pH}^{*} 9.0\right)$ containing $25 \% \mathrm{ACN}$.

\section{Results and Discussion}

Characterization of NRAuNPs. In a previous study, we demonstrated that NRAuNPs fluoresce weakly as a result of FRET and collisions. ${ }^{33} \mathrm{NR}$ contains an electrondonor diethylamino group and displaces citrate ions from the AuNP surface without altering the particle morphology. Because the fluorescence intensity of NR molecules becomes a function of its distance from the metal surface, the fluorescence of NR molecules in direct contact with the metal is completely quenched. ${ }^{38}$ The fluorescence of NRAuNP solutions is also very weak in the presence of BSA because it does not displace the NR product from the AuNP surface. We note that 32-nm NRAuNPs treated with BSA are stable in high-salt media; only very slight aggregation occurs, as indicated by the color photograph of an optical dark-field scattering image (Figure 2A). The SPR band of BSA adsorbed NRAuNPs that was measured by using a commercial UV-vis absorption spectrometer changed only slightly in high salts, supporting the result displayed in Figure 2A. Owing to diffusion and low scattering intensity of the $32-\mathrm{nm}$ NPAuNPs, the scattering light from single NPAuNPs (green spots) is not easily observed by our system. It is important to highlight that the red shift of scattering occurs once the NRAuNPs aggregate; the orange and red spots correspond to the scattering images of the NRAuNP aggregates. The optical images of the NRAuNPs are larger than their actual sizes because the optical diffraction limit $(\sim 400 \mathrm{~nm})$ is greater than the size of the NRAuNPs. ${ }^{39}$ Figure $2 \mathrm{~B}$ reveals that the NRAuNPs significantly aggregate in the presence of $1 \mathrm{mM}$ GSH, which is an observation that is in good agreement with our previous result. ${ }^{33}$ We suggest that

(38) Ditlbacher, H.; Krenn, J. R.; Felidj, N.; Lamprecht, B.; Schider G.; Salerno, M.; Leitner, A.; Aussenegg, F. R. Appl. Phys. Lett. 2002 $80,404-406$ 1062 
hydrophobic interactions and hydrogen bonding between the GSH-adsorbed AuNPs are responsible for aggregation. The fluorescence of the NRAuNPs is quite weak as result of FRET and the collisions depicted in Figure 2C, but the fluorescence increases (Figure 2D) once displacement of the NR product by GSH (1 mM) takes place. The results illustrated in Figure 2 suggest that the NRAuNPs can be used to determine the concentration of GSH and that the aggregation of AuNPs can be monitored by conducting scattering measurements.

To demonstrate the selectivity of NRAuNPs toward thiols, samples $(1.9 \mathrm{~mL})$ of $10 \mu \mathrm{M}$ NR and $1 \times$ NRAuNPs (both prepared in PBS solution) were added separately to $2 \mathrm{mM}$ hemoglobulin $(0.1 \mathrm{~mL})$, which is the most abundant protein in erythrocytes. ${ }^{40}$ It is not surprising that the fluorescence intensity of the NR solution at $654 \mathrm{~nm}$ increased slightly as a result of the formation of NRhemoglobulin complexes through hydrophobic interactions. On the other hand, the fluorescence intensity of the NRAuNP solution remained almost constant in the presence of hemoglobulin as a result of FRET, suggesting that the NR product-hemoglobulin complexes are adsorbed on the AuNP surface. Similar phenomena occur in the presence of $1 \mathrm{mM}$ carbonic anhydrase and $1 \mathrm{mM}$ asparagine. Thus, we expected a low fluorescence background to exist when using the NRAuNPs to explore single erythrocytes because the concentrations of hemoglobulin, carbonic anhydrase, and asparagine are higher than those found in single erythrocytes $\left(5.40 \times 10^{-4}, 8.05 \times 10^{-5}\right.$, and $3.72 \times 10^{-4} \mathrm{M}$, respectively). ${ }^{41}$ These results also suggest that NRAuNPs are suitable for measuring the GSH level in erythrocytes. Although we demonstrated the nonselective displacement of the NR product by other thiol compounds in our previous study, ${ }^{33}$ the intracellular concentration of GSH $(1-10 \mathrm{mM})$ in erythrocytes is relatively high when compared to that of other thiols. Its displacement rate is also much slower than those of other thiols, such as cysteine and homocysteine, which allows interference to be minimized by recording the background 20 min after the addition of the NRAuNPs to the cells (see the discussion below). A slow displacement reaction for GSH is due to its relative bulk, which is supported by the suggestion that the reaction rate of AuNPs with GSH is at least 5 orders of magnitude slower than that with homocysteine. ${ }^{31}$ Taking these factors together, we expected the interference from other thiols to be small $(<2 \%)$ and, thus, the NRAuNPs should be highly selective for the analysis of GSH in erythrocytes.

Comparison of NRAuNPs and NDA for the Determination of GSH Using CE-LIF. NDA and MBB are common reagents for precolumn labeling of thiols, such as GSH and cysteine, in CE. ${ }^{42}$ Hogan et al. demonstrated the determination of intracellular GSH in single erythrocytes by CE-LIF using MBB. ${ }^{15}$ To compare the sensitivity of NRAuNPs and NDA for the determination of GSH, we conducted CE-LIF in $10 \mathrm{mM}$ sodium tetraborate $\left(\mathrm{pH}^{*}\right.$ 9.0) containing $25 \%$ ACN. The fluorescent product of NDA with GSH was detected, whereas the NR product was detected in the case when NRAuNPs were used. We note that it was essential to add ACN to the background electrolyte to minimize the adsorption of the dyes and their complexes on the capillary wall. Table 1 lists the LOD, linearity, and precision for the analysis of GSH. The LODs for GSH at a signal-to-noise (S/N) ratio

(40) Hogan, B. L.; Yeung, E. S. Anal. Chem. 1992, 64, 2841-2845. (41) Dong, Q.; Wang, X.; Zhu, L.; Jin, W. J. Chromatogr. A 2002, 959, 269-279.

(42) Bayle, C.; Caussé, E.; Couderc, F. Electrophoresis 2004, 25, 14571472 .
Table 1. Determination of GSH in Erythrocytes through CE-LIF Using Various Fluorescent Dyes

\begin{tabular}{lccrcl}
\hline & $\begin{array}{c}\text { migration } \\
\text { time (min) } \\
(\mathrm{RSD}, n=3)\end{array}$ & $R^{2}$ & $\begin{array}{c}\text { LOD } \\
(\mathrm{nM})\end{array}$ & $\begin{array}{c}\text { GSH (mM) } \\
(\mathrm{RSD})\end{array}$ & ref \\
\hline $\mathrm{MBB}^{b}$ & $<10$ & $0.994^{a}$ & 48 & $2.20(18.6 \%)$ & 15 \\
$\mathrm{NDA}^{b}$ & $3.55(0.9 \%)$ & $0.999^{a}$ & 120 & $1.04(5.0 \%)$ & this study \\
$\mathrm{NRAuNPs}^{c}$ & $3.40(1.1 \%)$ & $0.984^{d}$ & 22 & $1.32(4.8 \%)$ & this study
\end{tabular}

a Correlation coefficients $\left(R^{2}\right)$ were calculated in the range of $1-100 \mu \mathrm{M} .{ }^{b} \mathrm{RBC}$ sample was pretreated with $10 \mathrm{mM}$ sodium tetraborate containing $80 \%$ ACN. ${ }^{c}$ RBC sample was prepared directly in $1 \times$ NRAuNP. ${ }^{d}$ Correlation coefficients were obtained in the range of $0.08-1 \mu \mathrm{M}$.

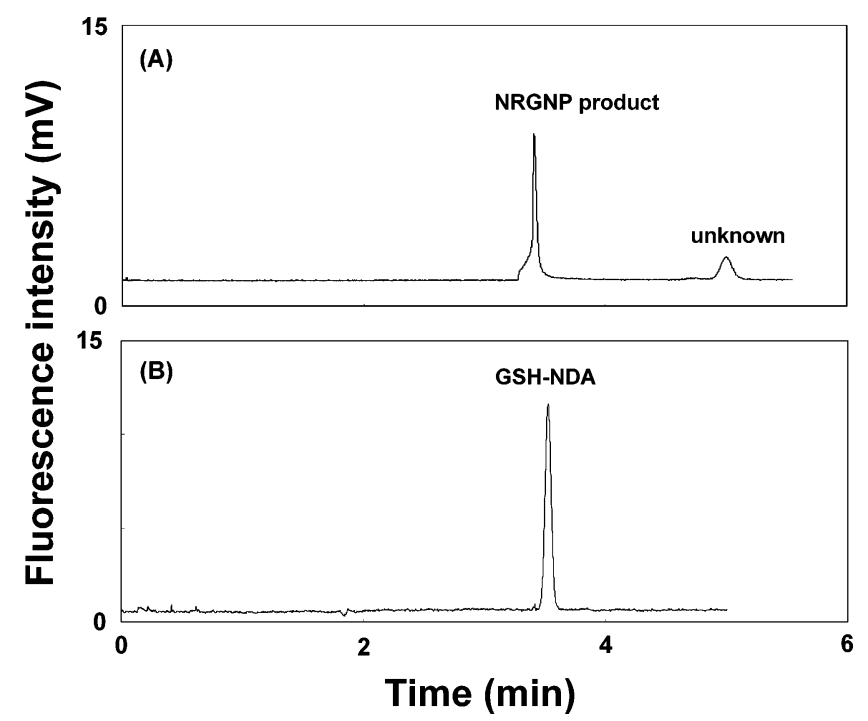

Figure 3. Electrophoretic separations of human erythrocyte lysates at $10 \mathrm{kV}$ using a $40-\mathrm{cm}$ capillary filled with $10 \mathrm{mM}$ sodium tetraborate $\left(\mathrm{pH}^{*} 9.0\right)$ containing $25 \%$ ACN. The effective length was $30 \mathrm{~cm}$. Erythrocytes were reacted with (A) $1 \times$ NRAuNPs and (B) $50 \mu \mathrm{M}$ NDA. The lysed samples were diluted (A) 2000-fold and (B) 20-fold prior to hydrodynamic injection (15-cm height for $15 \mathrm{~s}$ ).

of 3 were 48,120 , and $22 \mathrm{nM}$ when using MBB, NDA, and NRAuNPs, respectively. These results suggest that using CE-LIF in conjunction with NRAuNPs has great potential for diagnostic purposes. In comparison with our previous results, ${ }^{33} \mathrm{CE}-\mathrm{LIF}$ using NRAuNPs exhibits better linearity and sensitivity for the determination of thiols because the NR products were separated from NR. Panels A and $\mathrm{B}$, respectively, of Figure 3 display analyses of lysed erythrocytes using NDA and NRAuNPs. The RSD values of the migration times for the NR product and the GSH complexes are 1.1 and $0.9 \%$ when using NRAuNPs and NDA, respectively. We note that the mean GSH concentration $(1.04 \mathrm{mM})$ in single erythrocytes obtained when using NDA is relatively low when compared with that obtained (1.32 mM) when using NRAuNPs. This relatively low GSH concentration is likely to be the result of the loss of GSH that occurs during the treatment of the erythrocytes with $80 \%$ ACN that is required to avoid any interference from proteins when using NDA. ${ }^{43}$ On the other hand, the GSH concentration obtained when using NRAuNPs is slightly higher because of the displacement of the NR product by other thiols, such as cysteine.

Fluorescence Microscopy Measurements. On the basis of the results reported above, we were encouraged that it might be possible to conduct fluorescence microscopy to monitor the uptake of NRAuNPs in single

(43) Caussé, E.; Issac, C.; Malatray, P.; Bayle, C.; Valdiguié, P.; Salvayre, R.; Couderc, F. J. Chromatogr. A 2000, 895, 173-178. 

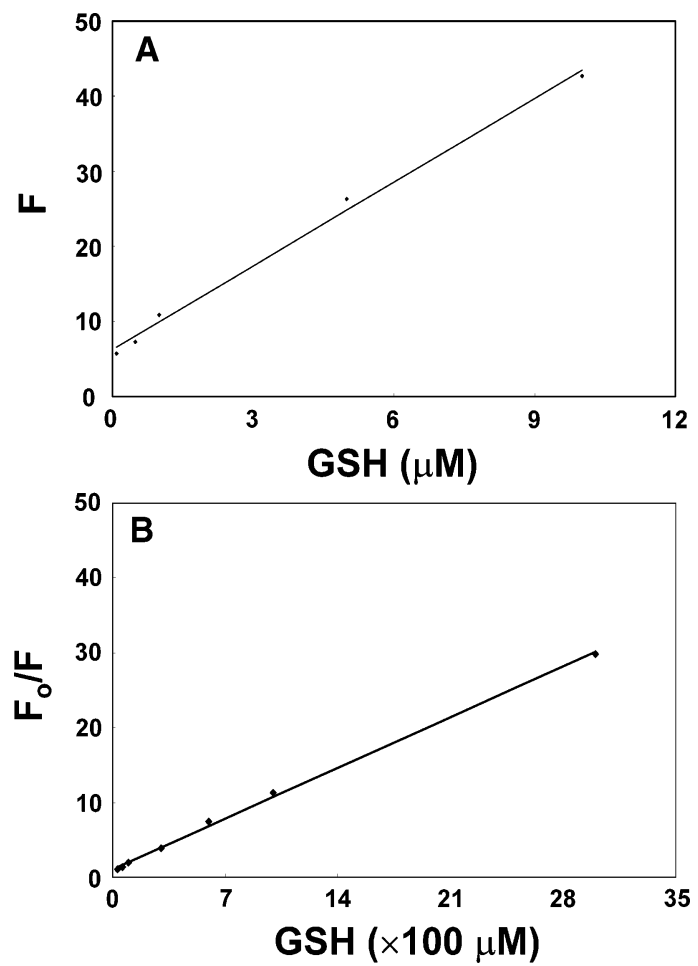

Figure 4. (A) Plot of $F$ (fluorescence intensity) versus GSH concentration within the range $0.1-10 \mu \mathrm{M}$; (B) plot of $\left[\left(F_{0} / F\right)\right]$ versus GSH concentration within the range 30-3000 $\mu \mathrm{M}$. The fluorescence was collected using a $100 \times$ objective and a 4-s exposure time.

erythrocyte cells. Prior to conducting such an analysis of erythrocyte cells, we used standard GSH solutions to test the sensitivity of the fluorescence microscopy system. After adding different amounts of GSH $(0.1-3000 \mu \mathrm{M})$ to the NRAuNPs and mixing for $10 \mathrm{~min}$, we dropped the solutions separately onto slides and immediately covered them with another slide to minimize the amount of evaporation. The fluorescence intensities of the solutions were then recorded using an inexpensive digital camera. We obtained a linear relationship ( $\left.R^{2}=0.9948\right)$ upon plotting of fluorescence intensity against the GSH concentration over the range from 0.1 to $10 \mu \mathrm{M}$ (Figure 4A). We note that the LOD for GSH is $\sim 10 \mathrm{nM}$ when the exposure time was set at $4 \mathrm{~s}$. Unexpectedly, we observed that the fluorescence intensity of the NR product decreased upon increasing the GSH concentration within the range of $30-3000 \mu \mathrm{M}$. Thus, the saturated GSH concentration is $\sim 30 \mu \mathrm{M}$, which corresponds to a surface coverage of $3.07 \times 10^{15}$ molecules of $\mathrm{GSH} / \mathrm{cm}^{2}$. At high GSH concentrations (>1 mM), the displacement of the NR product was saturated and serious aggregation of the NRAuNPs occurred as a result of hydrogen bonding between GSH molecules. ${ }^{31}$ The fluorescence was not saturated, but decreased upon increasing the GSH concentration. This is as a result of quenching by the AuNP aggregates. ${ }^{44}$ Greater extents of the NR products were trapped inside and adsorbed on the larger AuNP aggregates once they quickly formed (aggregation occurs more quickly at higher GSH concentration). Surprisingly, the plot depicted in Figure 4B exhibits good linearity $\left(R^{2}=0.9985\right)$ between the fluorescence intensity ratios $\left(F_{0} / F\right)$ and the GSH concentrations $(30-3000 \mu \mathrm{M})$. The value for the Stern-Volmer quenching constant is $0.0969 \mathrm{M}^{-1}$. The linear plot suggests that the proposed technique should allow the concentration of GSH to be

(44) Levi, S. A.; Mourran, A.; Spatz, J. P.; van Veggel, F. C. J. M.; Reinhoudt, D. N.; Möller, M. Chem. Eur. J. 2002, 8, 3808-3814. determined inside cells. To test this hypothesis, we loaded NRAuNPs into erythrocyte cells and monitored the changes in fluorescence by focusing a laser beam onto the whole cell (diameter of focusing area $=10 \mu \mathrm{m}$ ). The fluorescence within the single erythrocyte cells was brighter than that outside the cell (image not shown). The weak fluorescence background in the medium arose mainly from the NRAuNPs and possibly from the displacement of the NR product from the cell matrixes. The differences between the fluorescence intensity within the cell from that of the medium (diameter of detection area $=10 \mu \mathrm{m}$ ) were $5.9,11.1$, and $23.6 \mathrm{EV}$ (exposure values) at exposure times of 1,2 , and $4 \mathrm{~s}$, respectively. For the sake of sensitivity, we set the exposure time at $4 \mathrm{~s}$ for the following measurements.

Klaus et al. demonstrated that silver particles having diameters of up to $200 \mathrm{~nm}$ can accumulate within Pseudomonas stutzeri AG259 cells. ${ }^{45} \mathrm{Xu}$ et al. reported that three strains of Pseudomonas aeruginosa could take up and efflux silver nanoparticles having sizes ranging up to $80 \mathrm{~nm}$; this size is 1 order of magnitude larger than the reported exclusion limit of the outer membrane. ${ }^{46}$ Thus, it is our strong belief that the increase in fluorescence (Figure 5) occurs as a result of the 32-nm NRAuNPs being transported through the inner and outer membranes of the cells and then reacting with the thiols. Figure 5 depicts real-time fluorescence measurements of intact single erythrocytes incorporating NRAuNPs. After a 20-min loading of the NRAuNPs, the fluorescence within single erythrocyte cells gradually increased upon increasing the reaction time; the fluorescence reached a plateau after 2 $\mathrm{h}$, which is much slower than the time $(3 \mathrm{~min})$ required to displace the NR products by GSH in free solution. This slower rate is likely to be the result of the limited number of channels in the cell membrane that allow penetration of the NRAuNPs and also the time it takes after penetration for the NRAuNPs to be transported near or in the center of the cell where GSH is present in a high concentration. A slow transportation rate is expected because the heavy and bulky NRAuNP particles travel within a cell with a greater viscosity than that in the bulk medium. In addition, the slow diffusion rates of GSH and the NR product within the cells also contribute to the long equilibrium time. We note that the NR product does not transport to the membrane and diffuse out of the cell for times $>12 \mathrm{~h}$. After a 12-h incubation time, we used the linear plot depicted in Figure $4 \mathrm{~B}$ to calculate the mean GSH concentration in five single erythrocyte cells as being $1.30 \pm 0.31 \mathrm{mM}(\mathrm{RSD}=23.9 \%$; Table 2$)$. This result is in good agreement with that obtained by CE-LIF when using the NRAuNPs. Although other thiols such as cysteine also displace the NR product from AuNPs, their interference is not significant because of much lower concentrations inside the cells.

To further support our arguments regarding the transportation of the NRAuNPs, we operated the microscope system in conjunction with a digital video camera (exposure time, $33 \mathrm{~ms}$; readout time, $83 \mathrm{~ms}$ ) in a dark-field configuration. Figure 6 displays a sequence of consecutive images that depict the transport process of the NRAuNPs in living erythrocytes cells after a 40-min incubation time. Instead of absorption onto the glass surface, the NRAuNPs moved short distances $(\sim 2.28 \mu \mathrm{m})$ between successive images ( $4 \mathrm{~s}$ ) as a result of Brownian motion. We note that the disappearance of the NRAuNPs is likely to be due to

(45) Klaus, T.; Joerger, R.; Olsson, E.; Granqvist, C. Proc. Natl. Acad. Sci. U.S.A. 1999, 96, 13611-13614.

(46) Xu, X.-H. N.; Chen, J.; Jeffers, R. B.; Kyriacou, S. Nano Lett. 2002, 2, 175-182. 

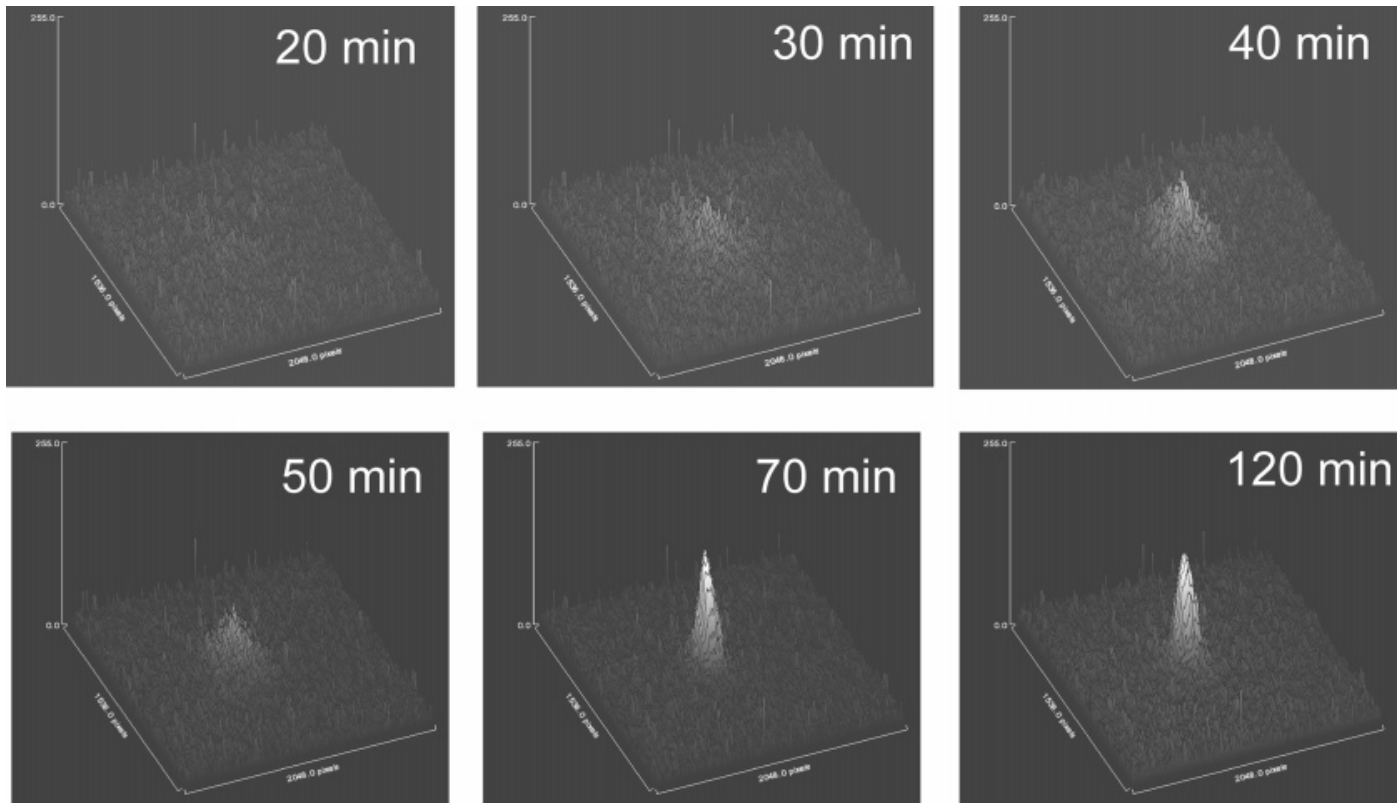

Figure 5. Real-time fluorescence images of the displacement of the NR product by GSH in erythrocytes. Time intervals were 5 min. The other conditions were the same as those described in Figure 4.

Table 2. Determination of GSH in Erythrocytes Labeled with Different Dyes by the Use of Fluorescence Microscopy Measurements

\begin{tabular}{|c|c|c|c|c|c|c|}
\hline & $\lambda_{\mathrm{ex}}(\mathrm{nm})$ & $\lambda_{\mathrm{em}}(\mathrm{nm})$ & laser & selectivity & $\begin{array}{l}\operatorname{variation}^{a}(\%) \\
(n=30)\end{array}$ & ref \\
\hline $\mathrm{MBB}^{b}$ & 380 & 490 & $\mathrm{He}-\mathrm{Cd}$ & thiols & 30 & 23 \\
\hline $\mathrm{NDA}^{c}$ & 472 & 528 & $\mathrm{Ar}^{+}$ & $\mathrm{GSH}, \gamma-\mathrm{GC}^{d}$ & 25.3 & this study \\
\hline $\mathrm{NRAuNPs}^{e}$ & 480 & 610 & $\mathrm{He}-\mathrm{Ne}$ or $\mathrm{Ar}^{+}$ & thiols & 23.9 & this study \\
\hline
\end{tabular}

${ }^{a}$ Cell to cell variation. ${ }^{b} 10 \mu \mathrm{M}$ MBB for $1 \mathrm{~h} .{ }^{c} 10 \mu \mathrm{M}$ for $10 \mathrm{~min} .{ }^{d} \gamma$-Glutamylcysteine. ${ }^{e} 1 \times$ NRAuNPs for $12 \mathrm{~h}$.

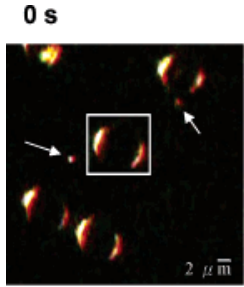

$4 \mathrm{~s}$
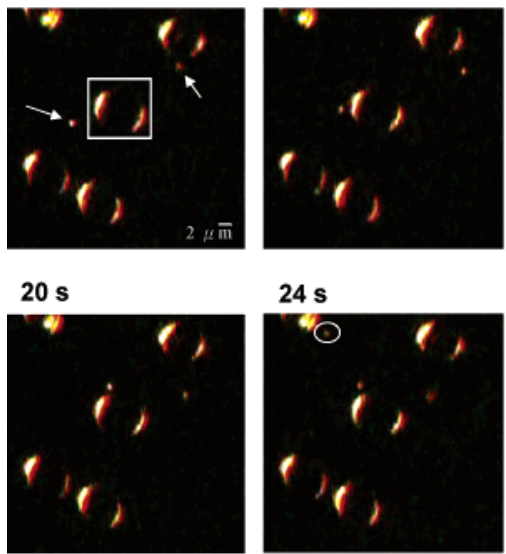

$40 \mathrm{~s}$
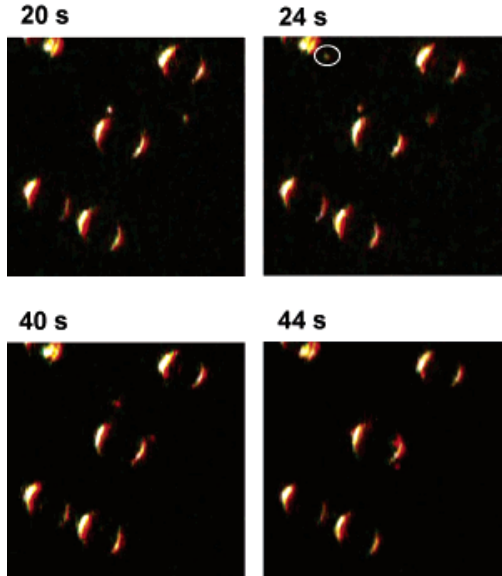

$44 \mathrm{~s}$

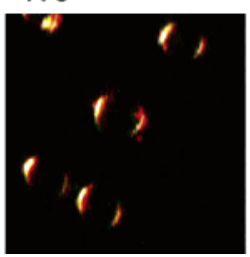

$8 \mathrm{~s}$

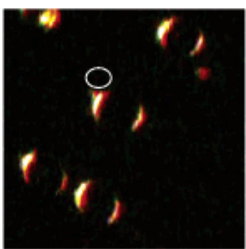

$12 \mathrm{~s}$
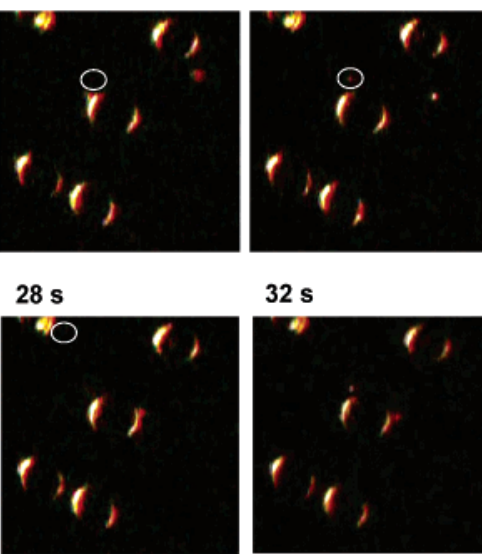

$48 \mathrm{~s}$

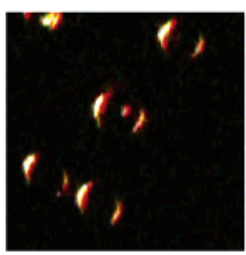

$32 \mathrm{~s}$

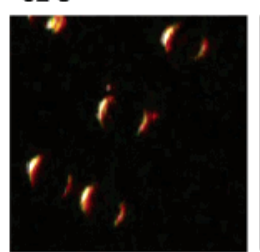

$52 \mathrm{~s}$

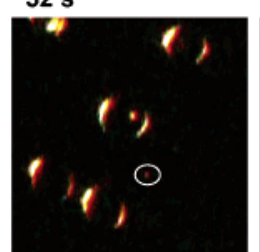

$16 \mathrm{~s}$

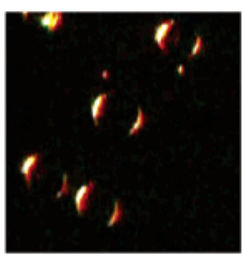

$36 \mathrm{~s}$

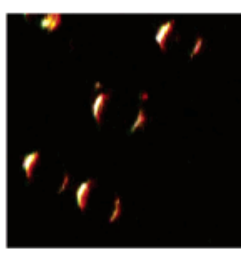

$56 \mathrm{~s}$

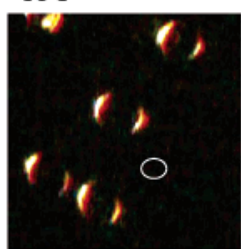

Figure 6. Consecutive scattering images displaying the process of transportation of $1 \times$ NRAuNPs in erythrocytes. The scattering images were recorded using a $40 \times$ objective with a $33-\mathrm{ms}$ exposure time (readout time $=83 \mathrm{~ms}$ ). The NRAuNPs and the target cell are marked with an arrowhead and a square, respectively.

uptake (e.g., at 8 and $44 \mathrm{~s}$ ) by erythrocyte cells or by their moving out of the focusing area (e.g., between 24 and 28 $\mathrm{s}$ and between 52 and $56 \mathrm{~s})$. The NRAuNPs tended to remain on the surface of the cell membranes for short periods of time before being transported through the cell membranes. As a result of interacting with membrane proteins and entering a more viscous environment, the NRAuNPs traveled more slowly within the cells than they did in the free solution. The diffusion rates of the NRAuNPs in the free solution and within the cell membrane were 0.57 (calculated from 0 to $8 \mathrm{~s}$ ) and 0.25 $\mu \mathrm{m}^{2} / \mathrm{s}$ (calculated from 48 to $52 \mathrm{~s}$ ), respectively. We note 

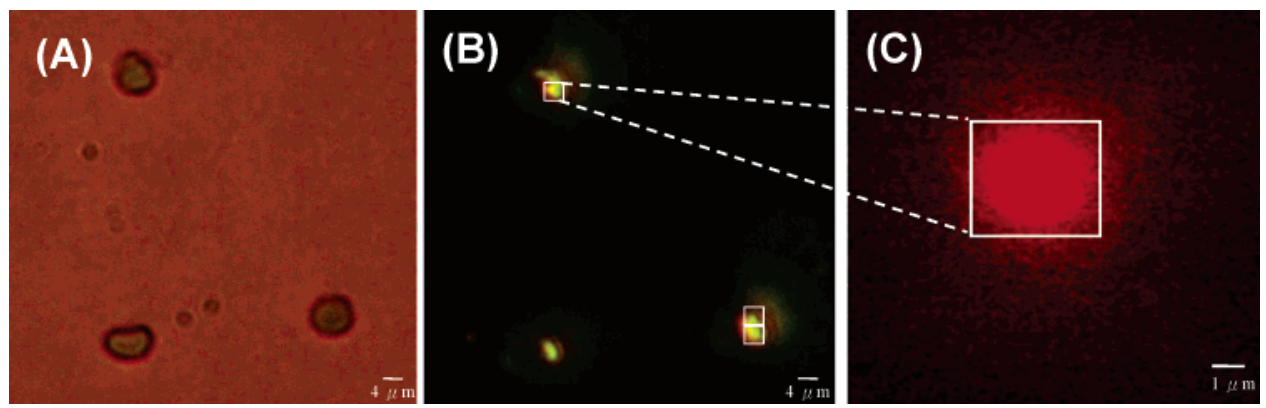

Figure 7. Comparison of the (A) phase contrast, (B) scattering, and (C) fluorescence images of erythrocytes incubated with $1 \times$ NRAuNPs for $12 \mathrm{~h}$. The exposure times were (A) 0.25 , (B) 0.5 , and (D) $4 \mathrm{~s}$, respectively. The light was collected using a $40 \times$ objective for the phase contrast and scattering measurements and a $100 \times$ objective for the fluorescence measurements.

(A)
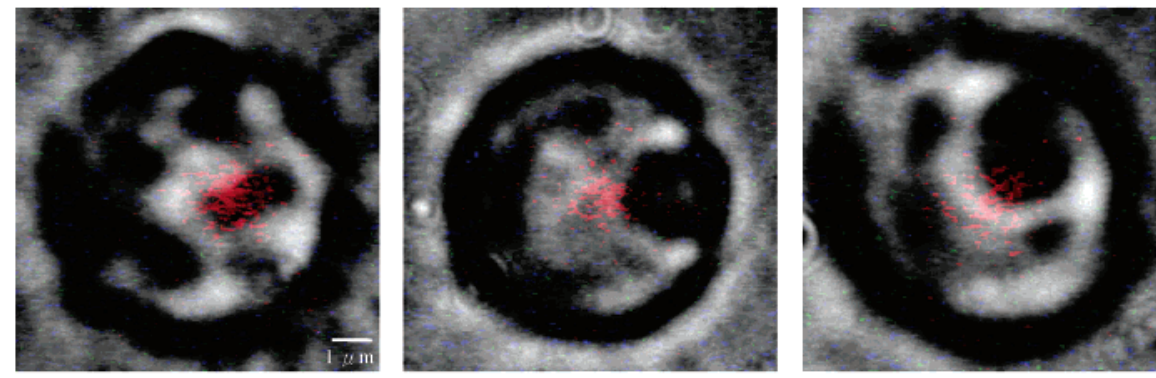

(B)
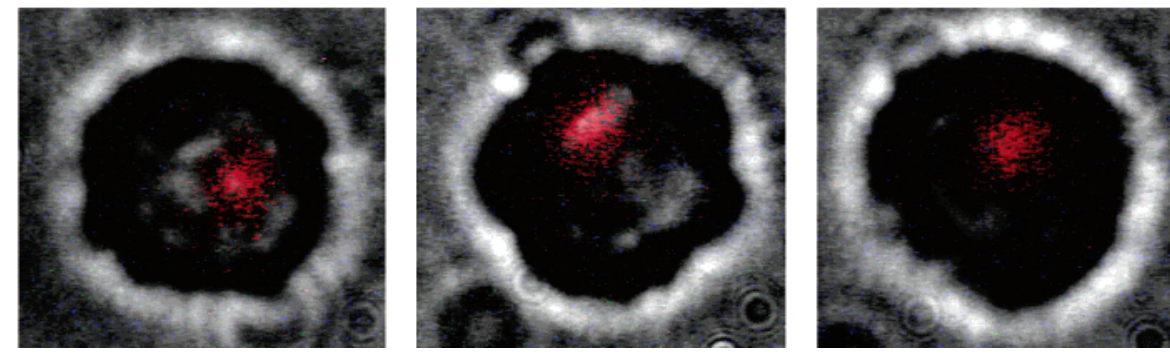

Figure 8. Images displaying the localization of GSH within erythrocytes. The living cells were incubated with (A) $1 \times$ NRAuNPs and (B) $10 \mu \mathrm{M}$ NDA for $12 \mathrm{~h}$ and $10 \mathrm{~min}$, respectively. Exposure time was $0.25 \mathrm{~s}$. The other conditions were the same as those described in Figure 5.

that various diffusion rates are expected for different sizes of NRAuNPs and for different environments.

We conducted dark-field scattering and fluorescence measurements to view the localization of NRAuNPs (GSH) in single erythrocytes after a 12 -h incubation time. Before conducting these measurements, we viewed the cells in the phase contrast mode, using the three erythrocyte cells indicated in Figure 7A. Representative scattering images of these three cells are presented in Figure 7B, in which the bright spots illustrate the presence of NRAuNPs within the cells. Because of poor spatial resolution and the small viewing area (limited by the laser light), the fluorescence image in a single erythrocyte cell was recorded for each measurement. Figure $7 \mathrm{C}$ presents one representative fluorescence image (the upper cell in Figure 7B), which indicates that the displacement and aggregation of NRAuNPs induced by GSH occur in a manner similar to the occurrence of those processes in free solution.

To indicate more clearly the localization of GSH within the erythrocytes through fluorescence measurements using NRAuNPs, we defocused the laser light in such a manner as to allow monitoring of a whole cell. In Figure 8A, the red images near the center of each of three different erythrocyte cells correspond to the GSH distributions. To confirm the localization of GSH within the cells, we incubated the erythrocytes with NDA, which is a highly selective fluorogenic reagent for $\mathrm{GSH}^{16}$ The images presented in Figure 8B confirm the distribution of GSH in erythrocytes and are consistent with those obtained using the NRAuNPs. These results suggested that NRAuNP has potential for the determination of thiols inside cells. The aggregation of the NRAuNPs obtained from the scattering measurements supports the results of these fluorescence measurements. The equilibration time, however, is much longer than that ( $\sim 10 \mathrm{~min})$ required when using NDA to label GSH within the cells. The spatial resolution of the system using NRAuNPs is slightly poorer than that using NDA, mainly because of diffusion of the NR products inside the cells for a longer period of time. We point out that diffusion of the NR product is small because the transportation of NRAuNPs from the bulk solution to the central area of the cells took $>11 \mathrm{~h}$. Despite the shortage, NRAuNPs have potential for greater sensitivity and universality for aminothiols when surface-enhanced Raman spectroscopy or mass spectroscopy is conducted (manuscript in preparation).

\section{Conclusion}

We have constructed a scattering and fluorescence microscopy system and used it in conjunction with a digital camera to sense GSH within erythrocytes using NRAuNPs. We obtained two calibration curves for GSH over the concentration ranges $0.1-30$ and $30-3000 \mu \mathrm{M}$, respectively. This system allows real-time monitoring of the 
transportation of NRAuNPs and changes in fluorescence, and it has the advantages of high sensitivity and low cost. The changes in fluorescence within the cells result from the displacement of the NR product from the surface of the AuNPs; these changes are supported by images recorded from scattering measurements. Using the NRAuNPs and NDA separately allowed us to confirm the localization of GSH within living cells. Our results also suggest that NRAuNPs hold great promise for use in biological imaging and biotechnology applications. We aim to apply this technique to an investigation of the distributions of GSH within normal and cancer cells and to explore the activity of enzymes, such as GSH peroxidase and glutathione transhydrogenase, within cells by monitoring changes in the concentrations of GSH. We believe that this relatively inexpensive scattering and fluorescence microscopy system will be useful also for monitoring biological events when using other biofunctional nanoparticles, such as quantum dots and fluorescent $\mathrm{SiO}_{2}$ nanoparticles.

\section{Abbreviations}

BSA, bovine serum albumin; CE, capillary electrophoresis; FRET, fluorescence resonance energy transfer; GSH, glutathione; AuNPs, gold nanoparticles; LIF, laserinduced fluorescence; MBB, monobromobimane; MCB, monochlorobimane; NDA, 2,3-naphthalenedicarboxaldehyde; NR, Nile Red; PBS, phosphate-buffered saline; SPR, surface plasmon resonance.

Acknowledgment. This study was supported by the National Science Council of Taiwan under Contracts NSC 93-2113-M-002-034 and NSC 93-2113-M-002-035. W.-L.T. is grateful to the National Science Council for his postdoctoral fellowship in the Department of Chemistry, National Taiwan University, funded under Contract NSC 92-2811-M-002-079.

LA0511034 